\title{
A Possible Spin-Fluctuation Mechanism in the Emergence of Superconductivity in a Region of Magnetic Instability, Using the Example of PuCoGa 5
}

\author{
A. A. Povzner, A. G. Volkov, and L. R. Kabirova \\ B.N. Yeltsin Ural Federal University, Yekaterinburg, 620002 Russia \\ e-mail:a.a.povzner@ustu.ru
}

\begin{abstract}
The spin-fluctuation superconductivity mechanism of strongly correlated $d-f$ electrons is considered within the Hubbard model. It is shown that the emergence of superconductivity is possible under conditions of strong spin anharmonicity. Evaluations of the superconductivity temperature in the model developed for the electron structure of $\mathrm{PuCoGa}_{5}$ agree with the experimental data.
\end{abstract}

DOI: $10.3103 / \mathrm{S} 1062873813100274$

\section{INTRODUCTION}

Strongly correlated $d$ and $f$ metals manifest anomalies in electron and magnetic properties, the nature of which remains the subject of debate [1]. Metals arranged in the crossover region that divides $d$ and $f$ metals into superconducting and magnetically ordered categories occupy a special place $[1,2]$. The assumption that the pairing of the electrons of this group of metals is due to phonons (in the context of BCS theory) does not agree with the experimental data on temperature $T_{\mathrm{C}}[1,3]$.

It was shown earlier in approximations of random phases [4] and self-consistent medium fields [5, 6] that the superconducting state of strongly correlated electrons with $d$ symmetry of the order parameter could be the result of a spin-fluctuation pairing mechanism that is enhanced when it approaches the point of magnetic instability. However, the anharmonicity of any spin subsystem must be taken into account near the point of magnetic transition. In addition, the electron subsystem of $f$ metals includes two interacting groups of strongly correlated electrons that were not included in $\mathrm{ab}$ initio LDA + U approaches. Let us examine the Hubbard multiband model, in which the intraatomic Coulomb and exchange interactions of $d$ and $f$ electrons are considered along with their band motion:

$$
H=H_{0}^{(l)}+H_{f f}+H_{d d}+H_{f d} .
$$

Here, $H_{0}^{(l)}$ is the Hamiltonian of noninteracting $s(p)$, $d$, or $f$ electron $(l=s(p), d, f) ; H_{f d}$ is the Hamiltonian of the intersite exchange interaction of $f$ and $d$ electrons, and $H_{f f}$ and $H_{d d}$ are the Hamiltonians of interaction of $f$ and $d$ electrons [7].

To analyze the conditions for the emergence of the superconducting phase due to the singlet pairing of $l$ electrons, let us consider the normal and anomalous
Green's functions $G_{k, \sigma}^{(l)}=Z^{-1} \operatorname{Sp}\left\{T_{\tau} a_{l, \vec{k}, \sigma}^{+} a_{l, \vec{k}, \sigma} \sigma(\beta)\right\}$ and $F_{k, l}^{(\uparrow \downarrow)}=\operatorname{Sp}\left\{T_{\tau} a_{l, \vec{k}, \uparrow}^{+} a_{l,-\vec{k}, \downarrow}^{+} \sigma(\beta)\right\} / \operatorname{Sp}\left\{T_{\tau} \sigma(\beta)\right\}$, where $\sigma(\beta)=T_{\tau} \exp \left\{-\int_{0}^{\beta} H(\tau) d \tau\right\}$ is the scattering matrix that determines statistical sum $Z=\operatorname{Sp}(\sigma(\beta)), H(\tau)$ is Hamiltonian (1) in the Matsubara interaction representation; and $a_{l, \vec{k}, \sigma}^{+}\left(a_{l, \vec{k}, \sigma}\right)$ is the birth (annihilation) operator of electrons $(l=d, f)$ with quasi-pulse $\vec{k}$ and spin quantum number $\sigma= \pm 1$.

Using Laplace transformations, we reduce the multiparticle problem under study to a problem on the electron motion in internal stochastic exchange $\left(\vec{\xi}_{l, v}\right)$ and charge $\left(\eta_{l, v}\right)$ fields fluctuating in space and time:

$$
\begin{gathered}
\sigma(\beta)=\iint T_{\tau} \exp \left\{-\beta U^{-1}\left[\sum_{v, l}\left(\left|\vec{\xi}_{l, v}\right|^{2}+\left|\eta_{l, v}\right|^{2}\right)\right.\right. \\
\left.\left.+\sum_{v, \mu} \sum_{l \neq l} A_{v, \mu} \vec{\xi}_{l, v} \vec{\xi}_{l, \mu}\right]-\beta H(V)\right\}(d \xi d \eta), \\
H(V)=\sum_{k, l, \sigma} \varepsilon_{k, l} a_{k, l, \sigma}^{+} a_{k, l, \sigma}+\sum_{k, q, l, \sigma} V_{q, l, \sigma} a_{k, l, \sigma}^{+} a_{k+q, l, \sigma} \\
+\sum_{k, q, l}\left(V_{q, l}^{(+)} a_{k, l, \downarrow}^{+} a_{k+q, l, \uparrow}+\text { h.c. }\right) .
\end{gathered}
$$

Here, $\quad V_{l, v}^{(+)}=\left(\xi_{l, v, x}+i \xi_{l, v, y}\right) / \sqrt{2}, \quad V_{q, l, \sigma}=$ $\left[U^{(l)} T\left(1+J_{q}^{(f d)} / U^{(l)}\right)\right]^{1 / 2}, \eta_{l, v}=T \int_{0}^{\beta} d \tau \sum_{v}(\ldots), \vec{\xi}_{l, v}=$ $\sum_{q} \mathrm{e}^{i q v} c_{q}^{(l)} \vec{\xi}_{l, q}, A_{q}=J_{q}^{(f d)} / \prod_{l\left(\neq l^{\prime}\right)}\left(1+J_{q}^{(f d)} \frac{2 U^{(l)}+U^{\left(l^{\prime}\right)}}{U^{(l)} U^{(l)}}\right)$, where $J_{\vec{q}}^{(f d)}$ is the Fourier image of the parameter of the intersite $f d$ interaction; $l=f, d, \gamma=x, y, z, q=\left(\vec{q}, \omega_{2 n}\right)$, 
$k=\left(\vec{k}, \omega_{2 n+1}\right), \omega_{2 n}$ and $\omega_{2 n+1}$ are the Matsubara frequencies of Bose and Fermi, and $U^{(l)}$ is the Hubbard constant.

Let us calculate statistical $\operatorname{sum} Z=\operatorname{Sp}(\mathrm{s}(\beta))$ by the saddle-point method over variables $\xi_{0, l}^{(\gamma)}, \eta_{l, q}, r_{l, q, \gamma}, \theta_{l, q, \gamma}$, $\left(\xi_{l, q, \gamma}=r_{l, q, \gamma} \exp \left(i \theta_{l, q, \gamma}\right)\right.$ for $\left.q \neq 0\right)$ (see [8]). In this procedure, we determine the most probable configuration of stochastic fields, which is characterized by the average value of the square of the exchange field at the site $\xi_{l}^{2}=\xi_{l, \perp}^{2}+\xi_{l, z}^{2}$, while the squares of the transverse $\left(\xi_{l, \perp}^{2}\right)$ and longitudinal $\left(\xi_{l, z}^{2}\right)$ components of the stochastic field are proportional (in the paramagnetic case) to the amplitudes of fluctuations of the corresponding components of spin density and are determined from saddle-point conditions [8].

Our calculations of anomalous functions $F_{k, l}^{(\uparrow \downarrow)}$ are similar to those in BCS theory. This can be seen from the structure of the effective Hamiltonian (2), in which stochastic exchange-charge fields $V_{l, v, \sigma}$ and $V_{l, v}^{( \pm)}$, which fluctuate near their most probable values, are figure instead of the operators of ion shifts. As a result, we derive the equations

$$
\begin{gathered}
F_{k, l}^{(\uparrow \downarrow)}=\xi_{l, \perp}^{2} G_{k, \uparrow}^{(0, l)} G_{-k, \downarrow}^{(l)} \sum_{q} F_{k+q, l}^{(\uparrow \downarrow)}+G_{k, \uparrow}^{(0, l)} F_{k, l}^{(\uparrow \downarrow)} \\
\times \sum_{q}\left\langle\left|\delta \xi_{l, q, \perp}\right|^{2}\right\rangle G_{k+q, \downarrow}^{(l)}-G_{k, \uparrow}^{(0, l)} G_{-k, \downarrow}^{(l)} \\
\times \sum_{q}\left(\left\langle\left|\delta \xi_{l, q, z}\right|^{2}\right\rangle+\left\langle\left\langle\left.\delta \eta_{l, q}\right|^{2}\right\rangle\right) F_{k+q, l}^{(\uparrow \downarrow)}\right. \\
G_{k, \sigma}^{(l)}=G_{k, \sigma}^{(0, l)}+\xi_{l, \perp}^{2} G_{k, \sigma}^{(0, l)} F_{k, \sigma}^{(\sigma \bar{\sigma})} \sum_{q}\left(F_{k+q, l}^{(\sigma \bar{\sigma})}\right)^{+} \\
+G_{k, \sigma}^{(0, l)}\left(F_{k, l}^{(\sigma \bar{\sigma})}\right)^{+} \sum_{q}\left\langle\left|\delta \xi_{l, q, \perp}\right|^{2}\right\rangle F_{k+q, l}^{(\sigma \bar{\sigma})} \\
-G_{k, \sigma}^{(0, l)} F_{k, l}^{(\sigma \bar{\sigma})} \sum_{q}\left(\left\langle\left|\delta \xi_{l, q, z}\right|^{2}\right\rangle+\left\langle\left|\delta \eta_{l, q}\right|^{2}\right\rangle\right)\left(F_{k+q, l}^{(\sigma \bar{\sigma})}\right)^{+} .
\end{gathered}
$$

$$
\chi_{\|}^{(l)}\left(\xi_{l}\right)=2 \prod_{\alpha= \pm 1} g_{0}^{(l)}\left(\mu-U^{(l)} n_{l} / 2+\alpha \xi_{l}\right) / \sum_{\alpha} g_{0}^{(l)}\left(\mu-U^{(l)} n_{l} / 2+\alpha \xi_{l}\right) .
$$

In this case, the SF amplitude in subsystems of $d$ and $f$ electrons is

$$
\xi_{l}^{2}=B^{(l)}\left(D^{(l)} T\right)^{2}\left\{1+4 \frac{J^{(f d) 2}}{U^{(f)} U^{(d)}} \frac{D^{(l)} D^{(l)}}{1-B^{(l)} D^{(l)} / B^{(l)} D^{(l)}}\left(2+\frac{B^{(l) 3} D^{(l) 2}}{B^{(l) 3} D^{(l) 2}}\right)\right\} .
$$

Here, $l \neq l, D^{(l)-1}=\left(1-U^{(l)} \chi_{\perp}^{(l)}\left(\xi_{l}\right)+\kappa_{l} \xi_{l, z}^{2}\right)$ is the inverse factor of the exchange amplification of susceptibility of $d$ or $f$ electrons; $J^{(f d)}$ is the uniform part of the intersite $f-d$ exchange interaction; and $B^{(l)}$ is the expansion coefficient of the Lindhard function of $l$ electrons over the frequency in the effective mass approximation.

Since any increase in SF amplitudes is limited by electroneutrality requirements, we can find the satura- 
$g_{0}(\varepsilon), \mathrm{eV}^{-1} \operatorname{spin}^{-1}$

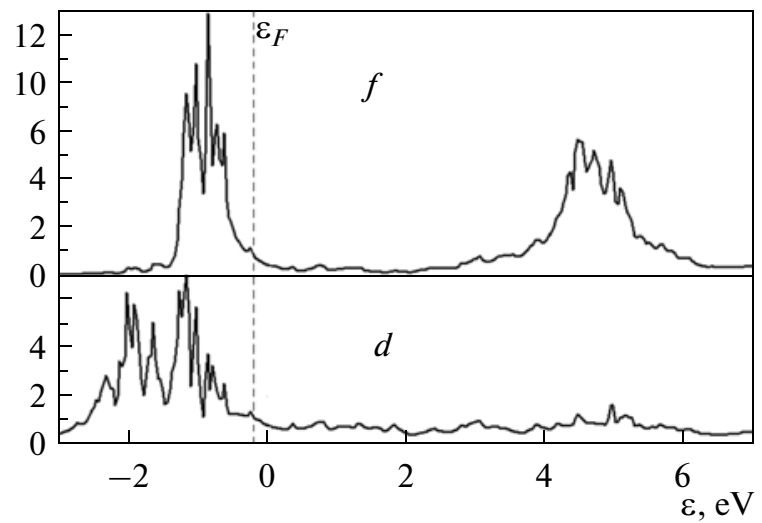

Fig. 1. Density of states of $f$ and $d$ electrons of $\mathrm{PuCoGa}_{5}$ at $U_{f}=2 \mathrm{eV}$ and $\xi_{l}=0$. The origin of energy consideration corresponds to the location of the Fermi level. The average distance between multiplet energies $\delta_{l}=2.5 \mathrm{eV}$.

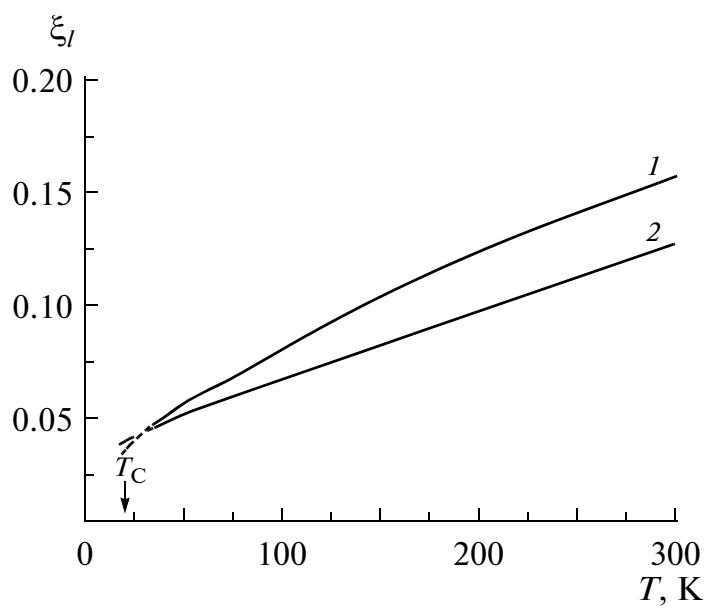

Fig. 2. Temperature dependence of the fluctuation amplitudes of spin density in the systems of $d$ and $f$ electrons of $\mathrm{PuCoGa}_{5}$, curves 1 and 2 , respectively; $\xi_{l}(l=d, f)$, calculated by formula (6) at $U_{f}=2 \mathrm{eV} ; U_{d}=1.6 \mathrm{eV}$; Hund exchange interaction $J_{H}=0.48 \mathrm{eV}$; and parameters of the imaginary part of the Lindhard function of $f$ and $d$ electrons $B^{(f)}=0.6, B^{(d)}=B^{(f)}\left(U^{(d)} g_{0}^{(d)}\left(\varepsilon_{F}\right) / U^{(f)} g_{0}^{(f)}\left(\varepsilon_{F}\right)\right)$.

tion temperatures of the SF amplitude $\left(T_{l}^{*}\right)$ for each subsystem. At $T \geq T_{l}^{*}$, longitudinal $\mathrm{SFs}$ disappear $\left(\chi_{\|}^{(l)}\left(\xi_{l}\right)=0\right)[7,8]$, while the spin rigidity coefficient is always positive.

Our analysis of instability in the formation of superconductivity in the $\mathrm{PuCoGa}_{5}$ normal phase was performed by self-consistent calculations $[9,10]$ of the electron structure in the LDA $+\mathrm{U}+\mathrm{SO}$ scheme (Fig. 1). These calculations were supplemented by calculations of the SF amplitude (Fig. 2) and magnetic susceptibility, the temperature dependence of which corresponded to the experimental data in [11] (to avoid considering $U^{(l)}$ twice, $n_{l}$ was replaced by the variation in temperature when filling the $l$ band). Our estimate of the correlation radii of the spin density of $f$ and dsubsystems $\left(R_{C}^{(l)}=\left(a_{l} D^{(l)}\right)^{1 / 2}\right.$, where $\left.a_{l}=0.1\right)$ is the parameter of spatial nonuniformity of the Lindhard function $[12,13])$ in the temperature region of instability caused by singlet pairing yields threefour interatomic distances.

According to our results, the chemical potential of $\mathrm{PuCoGa}_{5}$ lies in the region of negative curvature in the energy dependences of the densities of $d$ and $f$ states, and $\left|\kappa^{(f)}\right|<\left|\kappa^{(d)}\right|$. Our solutions to Eq. (4) for $d$ electrons correspond to higher temperatures. The value of $T_{\mathrm{C}}$ of $\mathrm{PuCoGa}_{5}$ calculated for $d$ electrons turns out to be $10 \mathrm{~K}$ at $J^{(f d)}=0$. The inclusion of $f, d$ interaction leads to the experimentally observed value of $18.5 \mathrm{~K}$ [11] at $J^{(f d)}=0.14 \mathrm{eV}$. An increase in $T_{\mathrm{C}}$ is possible as $J^{(f d)}$ grows. For example, at $J^{(f d)}=0.26 \mathrm{eV}, T_{\mathrm{C}}=42 \mathrm{~K}$.

\section{CONCLUSIONS}

The spin-fluctuation mechanism of singlet pairing of strongly correlated $f$ and $d$ electrons is observed in the negative curvature segment of the density of states near the Fermi level, particularly in features like minima. Interband interaction also promotes instability caused by the emergence of superconductivity. A strong dependence of temperature $T_{\mathrm{C}}$ on the electron concentration follows from the derived formulas, indicating the importance of experimental investigations of doped systems, which offer the possibility of controlling variations in concentrations of $d$ or $f$ electrons.

\section{ACKNOWLEDGMENTS}

This study was supported by the Ministry of Education and Science of the Russian Federation, contract no. 14.A18.21.0737.

\section{REFERENCES}

1. Moore, K.T. and van der Laan, G., Rev. Mod. Phys., 2009, vol. 81, p. 235.

2. Boring, A.M. and Smith, J.L., Challenges in Plutonium Science, Los Alamos: Los Alamos Sci., 2000, vol. 1.

3. Piekarz, P., Parlinski, K., Jochym, P.T., et al., Phys. Rev. B, 2005, vol. 72, p. 014521.

4. Qi, X.-L., Raghu, S., Liu, C.-X., et al., arXiv: 0804.4332v2. 
5. Berk, N.F. and Schrieffer, J.R., Phys. Rev. Lett., 1966, vol. 17, p. 433.

6. Chong, S.V., Mochiji, T., and Kadowaki, K., J. Phys.: Conf. Ser., 2009, vol. 150, p. 052036.

7. Moriya, T., Spin Fluctuations in Itinerant Electron Magnetism, Berlin: Springer-Verlag, 1985.

8. Volkov, A.G., Povzner, A.A., Kryuk, V.V., et al., Fiz. Tverd. Tela, 1999, vol. 41, p. 1793.

9. Povzner, A.A., Volkov, A.G., and Filanovich, A.N., Fiz. Tverd. Tela, 2010, vol. 52, p. 1879.
10. Povzner, A.A., Volkov, A.G., and Filanovich, A.N., Pis'ma Zh. Tekh. Fiz., 2011, vol. 36, p. 47.

11. Sarrao, J.L., Morales, L.A., Thompson, J.D., et al., Nature, 2002, vol. 420, p. 297.

12. Dzyaloshinskii, I.E. and Kondratenko, P.S., Zh. Eksp. Teor. Fiz., 1976, vol. 70, no. 5, p. 1987.

13. Moriya, T., Usp. Fiz. Nauk, 1981, vol. 135, p. 117.

Translated by N. Korovin 